\title{
TRAIN COLLISION AVOIDANCE WITH MOBILE DEVICES FOR SPARSELY USED RAILWAY LINES
}

\author{
THOMAS STRANG ${ }^{1,2}$, ANDREAS LEHNER ${ }^{1,2} \&$ BENNO KÜHNL $^{2}$ \\ ${ }^{1}$ German Aerospace Center (DLR), Institute of Communications and Navigation, Germany \\ ${ }^{2}$ Intelligence on Wheels (IoW) GmbH, Germany
}

\begin{abstract}
This paper shows the results of a communication range measurement campaign performed with mobile onboard units of a fully de-centralized train collision avoidance safety overlay system in typical environments of sparsely used railway lines. The most critical sections of the track with respect to a direct train-to-train communication channel were identified and selected for measurements in different antenna configurations. The measurement results justify the conclusion that the direct train-to-train communication channel along typical sparsely used railway lines fits the requirements of mobile onboard units of the train collision avoidance system.
\end{abstract}

Keywords: TrainCAS, TETRA, collision avoidance, safety, railway, trains, NGT.

\section{INTRODUCTION}

Although rail transport is extremely safe, collisions of railway vehicles happen occasionally. The safety overlay system described here adopts a very successful concept from aeronautics for avoiding the collision of trains [1]. It combines three core technologies: A direct train-totrain communication system, an accurate localization system, and a cooperative situation analysis and decision support system. Each equipped train determines its track-selective position on the line as well as other relevant parameters such as the current calculated braking distance, and broadcasts them per direct train-to-train communication to all other trains in communication range. Intelligent algorithms on board the receiving trains evaluate this information and raise an alarm in case of danger.

Acting as independent overlay system there is no need for a $100 \%$ penetration rate before being able to take advantage of the additional safety provided through the system. It does intentionally not interface with any other railway signalling technology which might or might not be in place or in operation along the line, to not inherit remaining failures or operational inadequacies. Thus it lifts the level of safety with every vehicle equipped with the technology. Despite being a technology to be used on rolling stock and relying on onboard technology only, it must be considered a fully de-centralized "virtual infrastructure", usually under the responsibility of the infrastructure management.

Whereas the best performance of the system can be achieved with fix mounted onboard technology, there are circumstances where operators prefer at least partially a mobile version of the technology which can be used only to temporarily equip vehicles on the track (see Fig. 1). This includes situations where there are additional guest or yellow fleet vehicles on the track, as well as situations when only a small varying set of vehicles out of a large vehicle fleet shall be used on a line.

One of the few restrictions of the mobile version compared to the fix mounted unit is the transmit power classes supported by the train-to-train transmitter hardware. Transmit power is a key factor determining the transmission range. Due to the large braking distances required for rolling stock once in movement, the transmission range defines the maximum speed at which the railway vehicles can be stopped early enough to avoid a potential collision. 


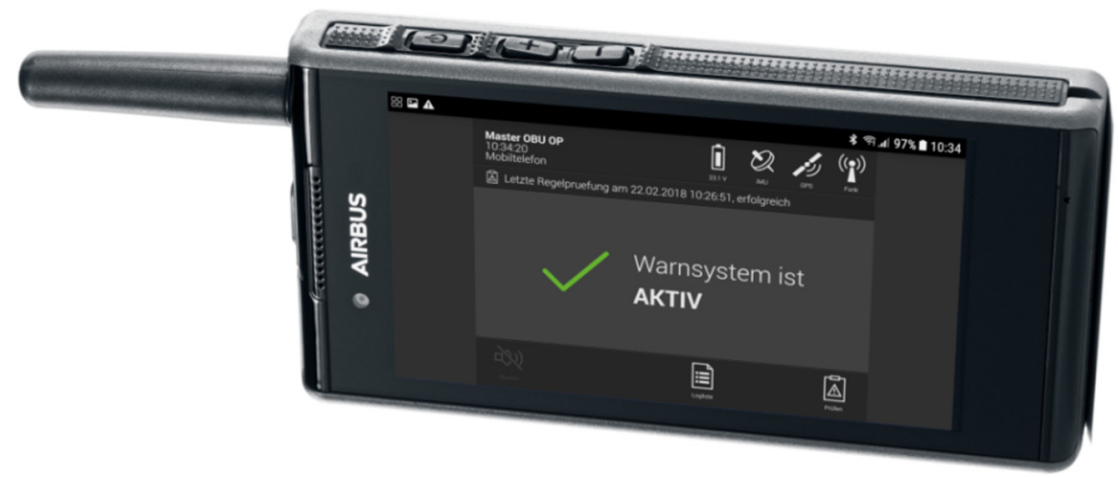

Figure 1: Mobile TrainCAS onboard unit.

This paper elaborates on the transmission ranges achievable with mobile units in typical sparsely used railway line environments.

\section{ANALYSIS}

Each mobile unit periodically broadcasts a status update containing position and braking distance information, utilizing a TETRA radio module using the short data service (SDS) in direct mode operation (DMO). The frequency is in the protected band of 380-430 MHz using EN300392-2 power class 3L, i.e. with up to $1.8 \mathrm{~W}$. Each single SDS received at a train's receiver is a successful communication between two equipped trains. The first successfully transmitted messages between approaching trains determine whether an upcoming critical situation can be avoided by raising an alarm timely enough.

The achievable communication range can be as large as almost $40 \mathrm{~km}$ with fix mounted onboard units in highspeed trains [2] using TETRA DMO-SDS without any base stations involved, well serving braking distances of several kilometres of trains running up to $300 \mathrm{~km} / \mathrm{h}$ each. It is unlikely that mobile onboard units will reach this communication range due to several reasons. One reason is the limited transmit power already mentioned. Another reason is the strong influence of the wave propagation channel [3] along urban and sub-urban railway lines with a variety of curves, reflectors (e.g. walls) and scatterers (e.g. trees). Finally, from an operational perspective the railway operators prefer to use the mobile units without any antennas on the roof of the trains, i.e. just with an antenna inside the driver cabin.

To assess the communication range of the mobile units in the aforementioned setup, a series of measurements were performed at typical sparsely used regional railway lines. In particular the most critical sections of the railway lines with respect to direct train-to-train communication range were identified and selected for the measurements. This encompassed for instance curved sections in forests and challenging sections through rural villages.

The measurements were performed with one mobile device acting as transmitter placed inside a train driver's cabin in a regional train during regular operations, running a service back and forth on a sparsely used regional line with one train per direction and hour. The antenna configuration was changed between runs to see differences. The communication peer was another onboard unit acting as receiver located in a road vehicle, stationary placed next to the track at the selected most critical sections of the line. At the receiver side two different antenna setups have been measured: One on the roof and one inside the vehicle. 


\section{RESULTS}

The measurement results - as expected - clearly show a difference whether a roof antenna was used at the receiver or not. For example, Fig. 2 (rural village) and Fig. 3 (timberline) show a minimum reception range of $2.7 \mathrm{~km}$ and $2.0 \mathrm{~km}$ respectively. Note that in all the figures the train with the transmitter was approaching the stationary receiver from one side, before passing and leaving it to the other side. Thus each figure illustrates the influence of the wave propagation channel on two sides, with the x-axis being normalized to the point in time of passing. Due to the strong influence of the channel it is very typical that the reception range of the two directions differ significantly, whereas the communication channel between the units is symmetric at any time, i.e. if receive and transmit functions are switched.

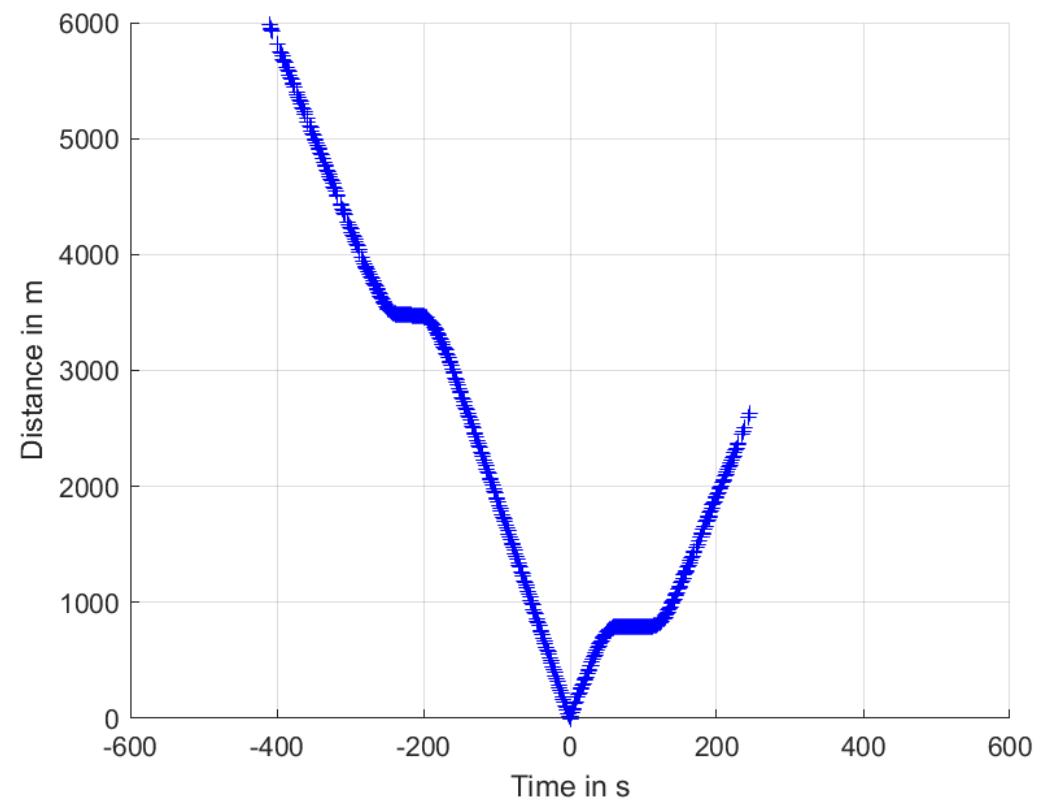

Figure 2: Reception ranges of train passing in rural village area (transmitter with internal antenna, receiver with roof antenna).

For the same scenarios (rural village, timberline), the results look different if no roof antenna is used at the receiver. Here the reception range degrades to $1.6 \mathrm{~km}$ (rural village) and $1.2 \mathrm{~km}$ (timberline) respectively as illustrated in Figs 4 and 5.

Despite the fact that the full potential of the use of an external antenna would be achieved if mounted on the roof of the vehicle, Figs 6 and 7 illustrate the potential of an external antenna used inside the driver's cabin of a train. Especially in the most demanding scenario (forest) with the reception range in Fig. 6 being slightly worse $(0.9 \mathrm{~km})$ compared to Fig. 5 (timberline), the use of an external antenna inside the driver's cabin can significantly improve the reception range to $2.4 \mathrm{~km}$ as seen in Fig. 7. 


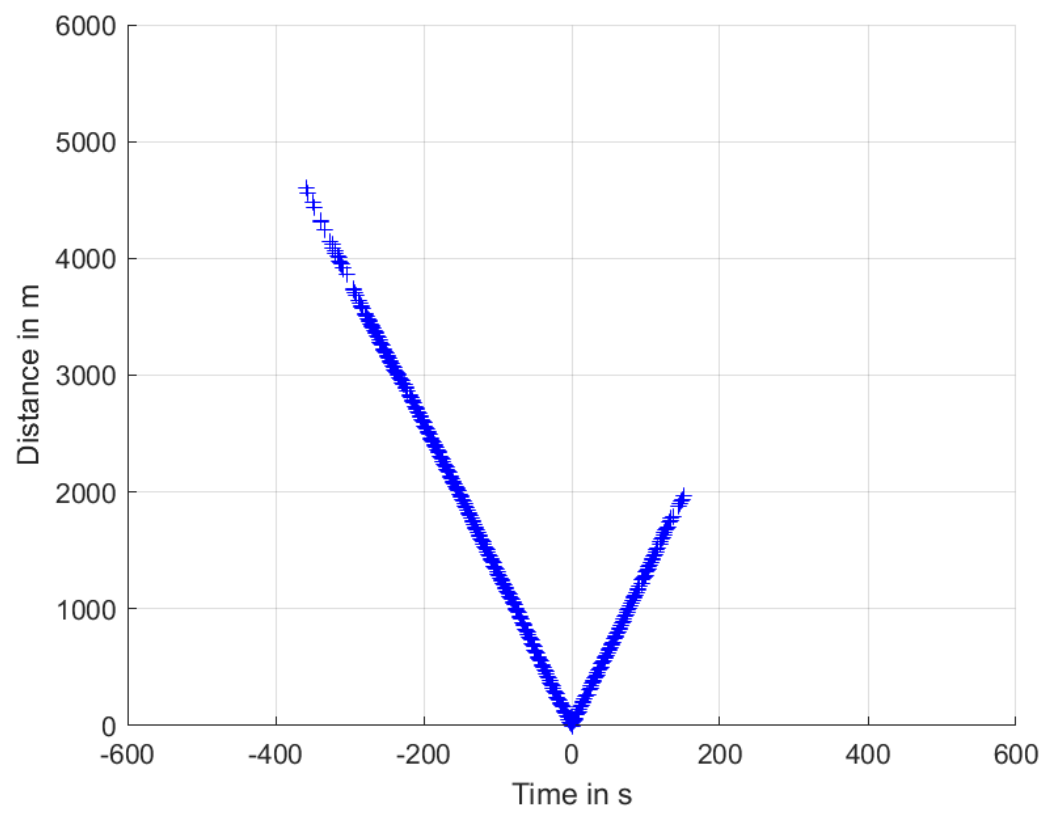

Figure 3: Reception ranges of train passing in timberline area (transmitter with internal antenna, receiver with roof antenna).

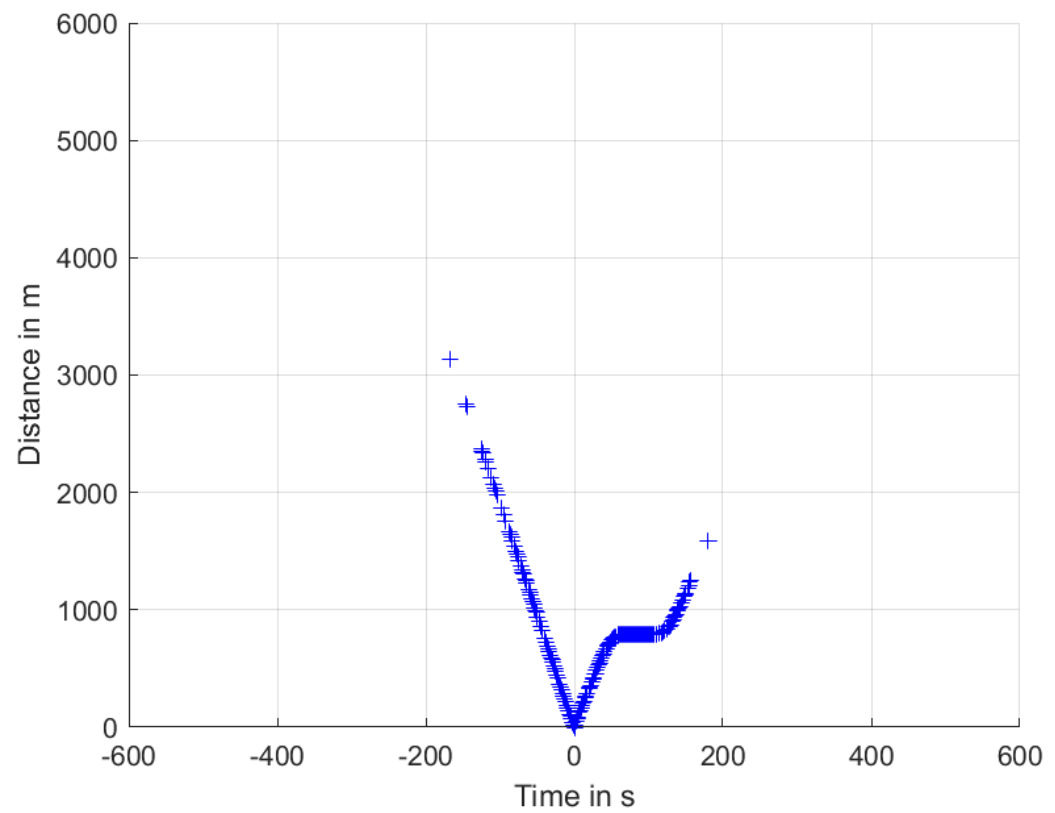

Figure 4: Reception ranges of train passing in rural village area (transmitter with internal antenna, receiver with internal antenna). 


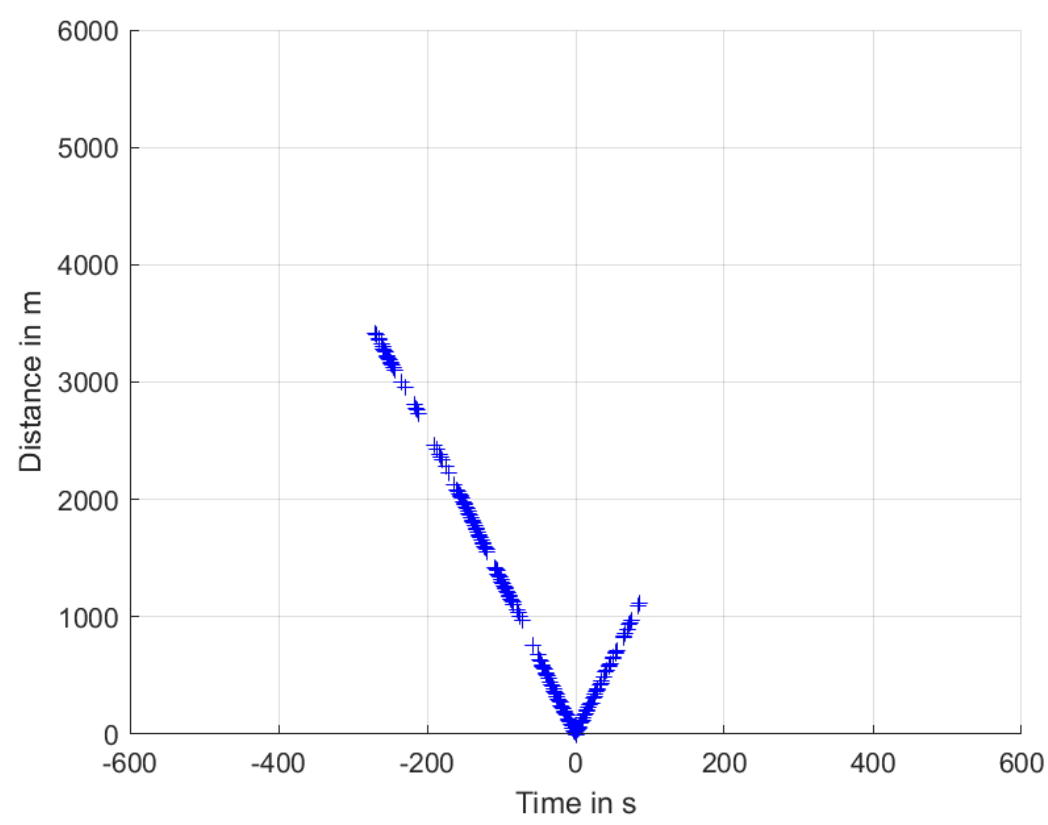

Figure 5: Reception ranges of train passing in timberline area (transmitter with internal antenna, receiver with internal antenna).

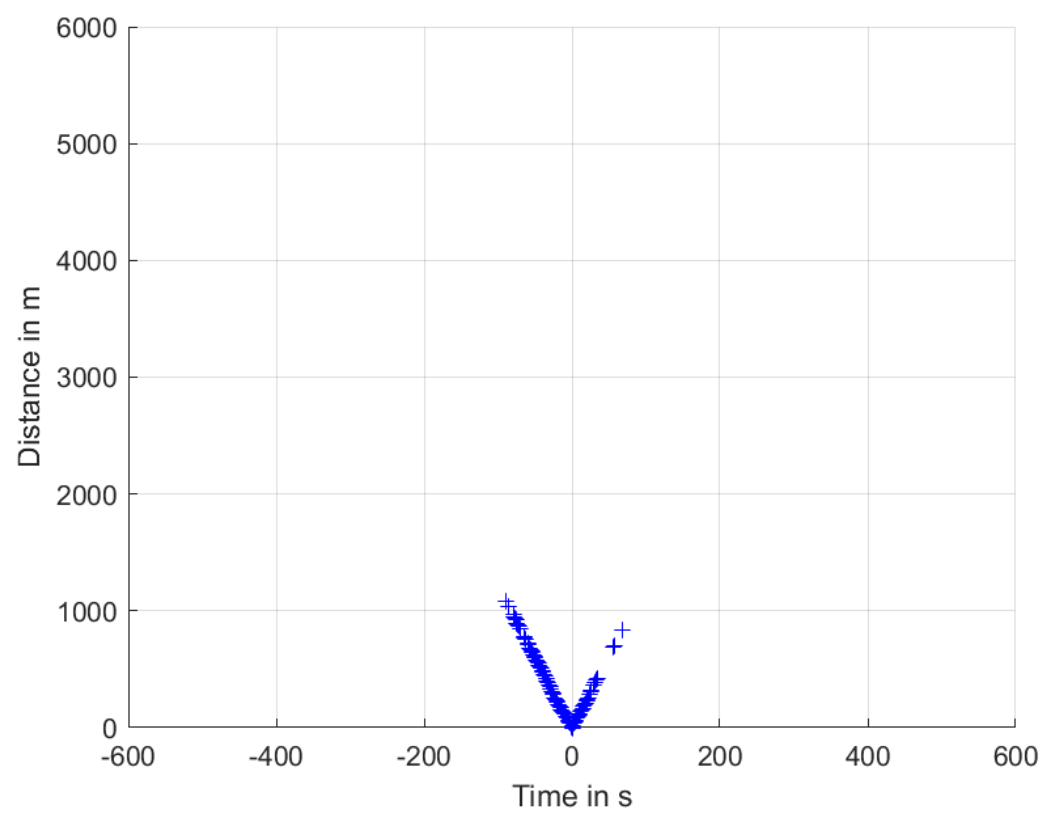

Figure 6: Reception ranges of train passing in forest area (transmitter with external antenna used inside driver's cabin, receiver with internal antenna). 


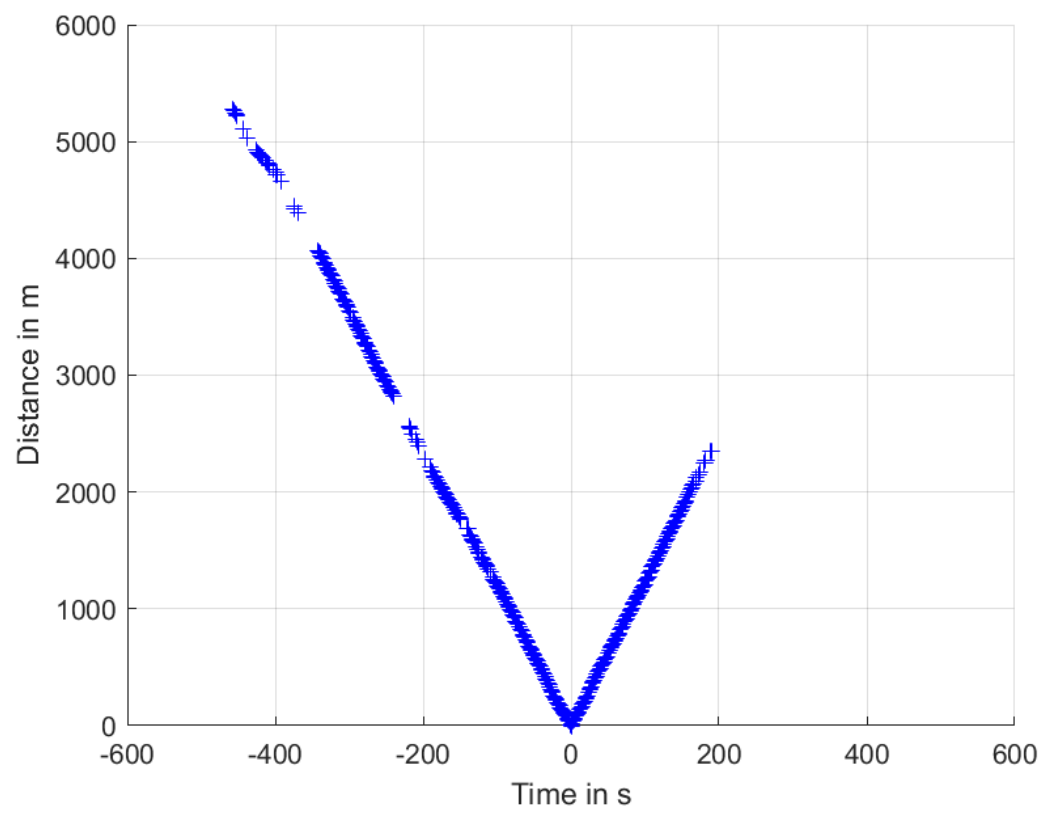

Figure 7: Reception ranges of train passing in forest area (transmitter with external antenna used inside driver's cabin, receiver with roof antenna).

\section{CONCLUSIONS}

Not surprisingly, the measurement results confirm the reduced communication ranges of TETRA DMO-SDS transmissions of mobile onboard units along typical sparsely used regional railway lines. Whereas a few kilometres of range can be achieved in less challenging parts of the railway network, the range can go down to about a kilometre or even less in challenging sections (environments), depending also on the used antennas and their position (inside driver cabin or on the roof).

For collision avoidance, the range has to be sufficiently large to be able to receive a message of an approaching train early enough to initiate a timely braking manoeuvre. This must include also any technical and human reaction times. The investigated railway line had a block size of $400 \mathrm{~m}$, i.e. all trains have to guarantee to be able to come to a complete standstill within $400 \mathrm{~m}$ - if necessary through an emergency braking manoeuvre - which is mainly ensured through a maximum speed limit to be obeyed along the line and a minimum braking ability of the train. That means for two opposite trains under protection of the collision avoidance overlay system, any reception range above $800 \mathrm{~m}$ would be sufficient. The collision avoidance system foresees an additional alert margin in the order of $15 \%$, which takes even larger variances in technical and human reaction times into account. This appears more than sufficient for instance for the operational circumstances experienced at the lines of the measurements, where a speed limit of $50 \mathrm{~km} / \mathrm{h}$ (equals $13.8 \mathrm{~m} / \mathrm{s}$ ) was to be obeyed by the train drivers. Thus there are $8 \mathrm{~s}$ of extra margin on the total reaction time after successful message reception even in the most critical sections along the line.

It should be further noted that the speed limits along the track vary from the general speed limit. Permanent speed restrictions are usually assigned to sections of the line with steep 
curves or other potential of danger. As there is high degree of overlap of those areas with areas of challenging communication propagation conditions, there is even more reaction time available.

To finally conclude, mobile onboard units are not negatively affected by the communication conditions along typical sparsely used railway lines from the collision avoidance application perspective. Whereas the device's internal antennas provide a performance already above the design boundary, the use of external antennas even inside the train can further improve the performance.

\section{ACKNOWLEDGEMENT}

This work was partly supported by the Federal Ministry for Economic Affairs and Energy on the basis of a decision by the German Bundestag.

\section{REFERENCES}

[1] Haas, K.-G., Better railway collision prevention with TrainCAS, the autonomous train collision avoidance system. Signal + Draht, 110, 2018.

[2] Lehner, A., Strang, T. \& Unterhuber, P., Direct train-to-train communications at low UHF frequencies. IET Microwaves, Antennas and Propagation, 12(4), pp. 486-491, 2017. DOI: 10.1049/iet-map.2017.0597.

[3] Rico Garcia, C., Lehner, A. \& Strang, T., Channel model for train to train communication using the $400 \mathrm{MHz}$ band. IEEE 67th Vehicular Technology Conference, Singapore, pp. 3082-3086, 2008. 